\title{
Energy of Midlatitude Transient Eddies in Idealized Simulations of Changed Climates
}

\author{
Paul A. O'Gorman* and Tapio Schneider \\ California Institute of Technology, Pasadena, California
}

(Manuscript received 14 June 2007, in final form 2 April 2008)

\begin{abstract}
As the climate changes, changes in static stability, meridional temperature gradients, and availability of moisture for latent heat release may exert competing effects on the energy of midlatitude transient eddies. This paper examines how the eddy kinetic energy in midlatitude baroclinic zones responds to changes in radiative forcing in simulations with an idealized moist general circulation model. In a series of simulations in which the optical thickness of the longwave absorber is varied over a wide range, the eddy kinetic energy has a maximum for a climate with mean temperature similar to that of present-day earth, with significantly smaller values both for warmer and for colder climates. In a series of simulations in which the meridional insolation gradient is varied, the eddy kinetic energy increases monotonically with insolation gradient. In both series of simulations, the eddy kinetic energy scales approximately linearly with the dry mean available potential energy averaged over the baroclinic zones. Changes in eddy kinetic energy can therefore be related to the changes in the atmospheric thermal structure that affect the mean available potential energy.
\end{abstract}

\section{Introduction}

How the position and strength of midlatitude storm tracks change as the climate changes is a fundamental question in atmospheric dynamics. Global warming scenarios consistently point to a poleward shift in stormtrack position, whose dynamical basis is not well understood (Yin 2005; Bengtsson et al. 2006). The nature of changes in storm-track strength, as measured, for example, in terms of wind speed or minimum pressure in cyclones, is less clear. Some studies point to an increase in intensity of cyclones with a decrease in the frequency of cyclones (e.g., Lambert 1995; Geng and Sugi 2003); others find little change in either statistic (Bengtsson et al. 2006). Yin (2005) finds an increase in transient eddy kinetic energy (EKE) in the Southern Hemisphere and different responses in summer and winter in the Northern Hemisphere.

Here we examine how the EKE in midlatitude baroclinic zones changes as the climate changes in two series

\footnotetext{
* Current affiliation: Massachusetts Institute of Technology, Cambridge, Massachusetts.
}

Corresponding author address: Paul O'Gorman, Department of Earth, Atmospheric, and Planetary Sciences, 54-1616, MIT, 77 Massachusetts Ave., Cambridge, MA 02139.

E-mail: pog@caltech.edu of simulations with an idealized moist general circulation model (GCM). We simulate a wide range of climates with statistically steady and axisymmetric circulation statistics and consider changes in climate resulting from changes in longwave optical thickness (representing changes in greenhouse gas concentrations) and changes in meridional insolation gradient (representing changes in, for example, high-latitude surface albedo). In addition to the total EKE, we also describe the behavior of the near-surface EKE to characterize the surface storminess in midlatitude storm tracks.

Several factors may exert competing effects on the energy of midlatitude eddies (Held 1993). Theories of dry baroclinic eddies suggest that eddy energies depend on the static stability and meridional temperature gradients in the atmosphere, either through the growth rate of linear baroclinic instability or through the mean available potential energy (MAPE; Lorenz 1955; Green 1970). Global warming simulations indicate that a climate warmer than that of present-day earth has a smaller near-surface meridional temperature gradient in the Northern Hemisphere (Meehl et al. 2007); this reduces baroclinic instability growth rates and MAPE and thus may lead to a reduction in EKE. On the other hand, the meridional temperature gradient in the upper troposphere increases in a warmer climate (Meehl et al. 2007); this increases baroclinic instability growth rates 
and MAPE and, which may lead to an increase in EKE. The dry static stability is expected to increase in a warmer climate, particularly in regions in which it is strongly influenced by moist convection (Korty and Schneider 2007), with a consequent decrease in baroclinic instability growth rates and MAPE and thus, possibly, a decrease in EKE.

Several previous studies have attempted to relate changes in EKE to changes in the lower-tropospheric Eady growth rate (e.g., Geng and Sugi 2003; Caballero and Langen 2005; Li and Battisti 2008) rather than the approach used here, which relates EKE to MAPE. Although MAPE and the lower-tropospheric Eady growth rate both depend on static stability and meridional temperature gradients, they do not in general behave in the same way because of the dependence of MAPE on the thermal structure of the upper troposphere.

In simulations with a dry idealized GCM, Schneider and Walker (2008) found that, over a wide range of climates, EKE scales linearly with MAPE averaged over baroclinic zones. Whether such results carry over to moist atmospheres depends on the role of latent heat release in the energetics of baroclinic eddies. Latent heat release can influence the growth of individual cyclones (e.g., Reed et al. 1988) and contributes to the budget of eddy available potential energy in the winter storm tracks (Chang et al. 2002). Latent heat release has also been found to increase growth rates in theoretical studies of moist baroclinic instability, (e.g. Emanuel et al. 1987), and it can increase the maximum EKE attained in the life cycle of baroclinic eddies $(\mathrm{Gu}-$ towski et al. 1992). For a zonal-mean state representative of present-day earth, Lorenz (1979) found that latent heat release leads to an increase of moist MAPE by approximately $30 \%$ over the dry MAPE, a difference that can be expected to increase in moister atmospheres. However, the difference between dry and moist MAPE found by Lorenz (1979) is largely due to contributions from low-level regions in the tropics that may not be important for midlatitude eddies. Furthermore, even if it is not the dry static stability that is important but rather an effective moist stability (Lapeyre and Held 2004), changes in the effective moist stability may still scale with changes in dry static stability in moist atmospheres. This would be the case if the effective moist stability is a weighted average of a dry stability and a much smaller moist stability in updrafts (cf. Emanuel et al. 1987), with the weighting coefficients (e.g., area fraction of updrafts and downdrafts) not changing significantly as the climate changes. It may therefore be possible to continue to relate changes in EKE to changes in dry MAPE even in a moist atmosphere. This is indeed the case in our simulations, allowing us to relate changes in EKE to changes in the mean thermal structure of the atmosphere.

\section{Model and series of simulations}

The idealized GCM is a hydrostatic primitive equation model with 30 vertical sigma levels and a horizontal resolution of T42; it is described in detail in O'Gorman and Schneider (2008). The insolation at the top of the atmosphere $\left(S_{\mathrm{TOA}}\right)$ is imposed as a perpetual equinox with no diurnal cycle,

$$
S_{\mathrm{TOA}}=\frac{S_{0}}{4}\left[1+\frac{\Delta_{s}}{4}\left(1-3 \sin ^{2} \phi\right)\right],
$$

where $\phi$ is latitude, $S_{0}=1360 \mathrm{~W} \mathrm{~m}^{-2}$, and the insolation gradient parameter $\Delta_{s}$ has a default value of 1.2. Solar radiation is absorbed in the atmosphere such that the downward shortwave flux at a given sigma level and latitude is

$$
S=S_{\mathrm{TOA}} \exp \left[-\tau_{s} \sigma^{2}\right],
$$

where $\tau_{s}=0.22$. The surface albedo is constant (0.38), with all reflected solar radiation escaping directly to space. A two-stream gray radiation scheme is used to parameterize longwave radiation. The longwave optical thickness is specified as $\tau=\alpha \tau_{\text {ref }}$, where the optical thickness of the reference simulation, $\tau_{\text {ref }}$, is a fixed function of pressure and latitude, and $\alpha$ is a rescaling parameter with default value $\alpha=1$ (O'Gorman and Schneider 2008). The reference simulation $(\alpha=1$, $\left.\Delta_{s}=1.2\right)$ has a climate similar to that of present-day earth. It has a global-mean total EKE of $0.6 \mathrm{MJ} \mathrm{m}^{-2}$, compared with an estimated $0.7 \mathrm{MJ} \mathrm{m}^{-2}$ for transient eddies in earth's atmosphere (Peixoto and Oort 1992).

In the first series of simulations, the longwave optical thickness is varied by setting the rescaling factor $\alpha$ to a different value in each of a series of 16 simulations $(\alpha=$ $0.2,0.4,0.6,0.7,0.8,0.9,1.0,1.2,1.4,1.6,1.8,2.0,2.5,3.0$, $4.0,6.0)$, with the insolation gradient parameter held fixed at $\Delta_{s}=1.2$. The changes in imposed longwave optical thickness should be taken as an idealized representation of changes in all longwave absorbers, including carbon dioxide and water vapor. The longwave optical thickness does not depend on the water vapor field, and so we are not allowing for radiative water vapor feedback or radiative effects of clouds. The hydrological cycle and extratropical thermal stratification in this series of simulations are investigated in O'Gorman and Schneider (2008) and Schneider and O'Gorman (2008).

In the second series of simulations, the meridional insolation gradient is varied by setting the parameter $\Delta_{s}$ to a different value in each of a series of 11 simulations 
a

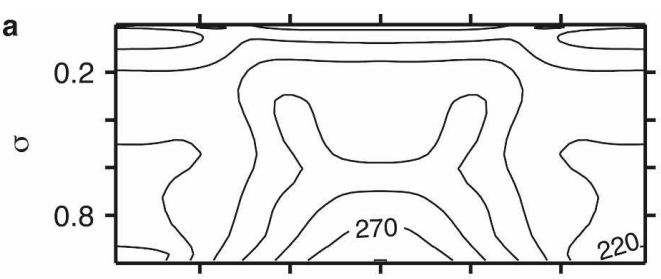

b

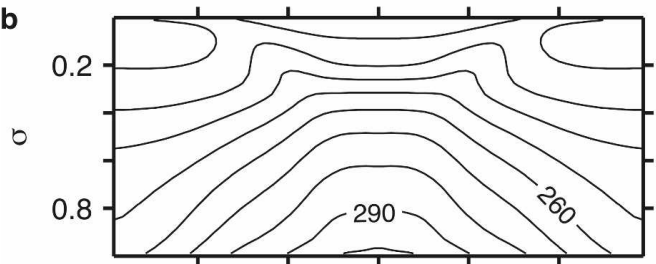

c

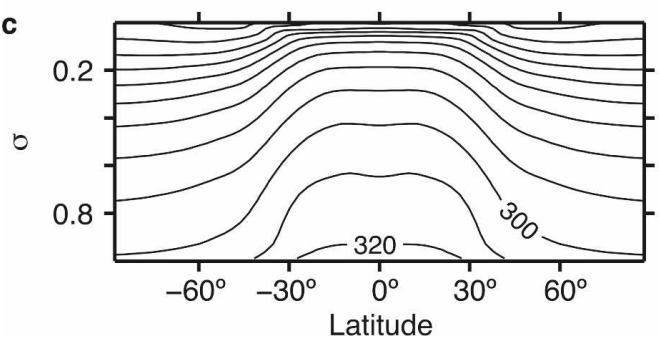

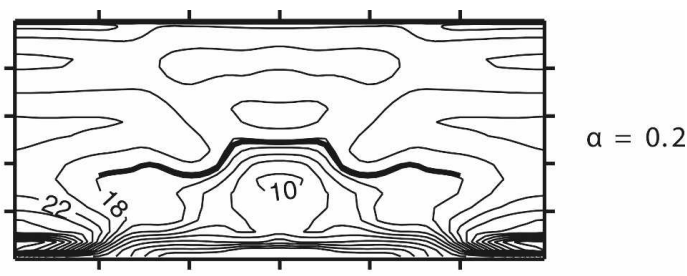
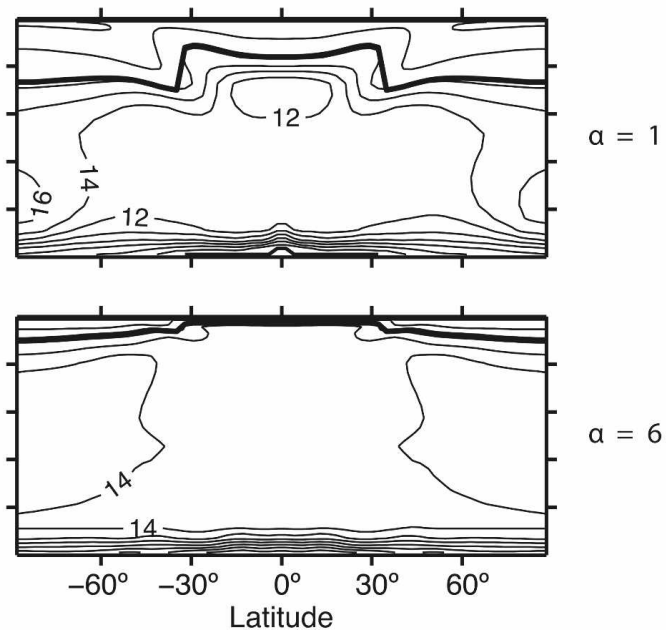

FIG. 1. Mean temperature and buoyancy frequency (zonal, time, and interhemispheric average) for simulations in the series in which the longwave optical thickness is varied $\left(\Delta_{\mathrm{s}}=1.2\right)$ : (a) coldest simulation $(\alpha=0.2),(\mathrm{b})$ reference simulation $(\alpha=1.0)$, and (c) warmest simulation $(\alpha=6)$. (left column) Temperature (contour interval $10 \mathrm{~K}$ ). (right column) Buoyancy frequency (contour interval $2 \times 10^{-3} \mathrm{~s}^{-1}$ ) and tropopause (thick solid line). The tropopause level is determined as a surface of temperature lapse rate $2 \mathrm{~K} \mathrm{~km}^{-1}$ and becomes poorly defined in high latitudes in the coldest simulation.

$\left(\Delta_{\mathrm{s}}=0.1,0.2,0.4,0.6,0.8,1.0,1.2,1.4,1.6,1.8,1.95\right)$, with the longwave optical thickness held fixed $(\alpha=1)$. Changes in the insolation gradient are used as a surrogate for changes in the distribution of shortwave forcing, such as might occur through changes in highlatitude surface albedo. Changes in $\Delta_{s}$ do not affect the global-mean insolation.

In taking effects of moisture into account, we only consider the vapor-liquid phase change and use a simplified form of the Clausius-Clapeyron relation with constant latent heat of vaporization. A simple quasiequilibrium scheme, a variant of that described in Frierson (2007), is used to parameterize moist convection by relaxation of temperatures toward a profile with a moist adiabatic lapse rate, and of specific humidities toward a profile with a reference relative humidity of $70 \%$. A grid-scale condensation scheme ensures that the relative humidity in a grid cell does not exceed $100 \%$, and falling condensate is assumed not to reevaporate. The effect of water vapor on the density of air (virtual temperature effect) is taken into account in the model. Evaporation at the lower boundary of the GCM, a mixed layer ocean, provides a moisture source for the atmosphere. See O'Gorman and Schneider
(2008) for other details of the model and of the series of simulations in which the longwave optical thickness is varied.

Because of the symmetries of the boundary conditions, statistics of the simulated climates are steady and zonally and hemispherically symmetric. The mean thermal structures of several of the simulations are shown in Figs. 1 and 2. Figure 1 shows how increases in longwave optical thickness $(\alpha)$ lead to increases in globalmean surface air temperature and tropopause height, with decreases in the pole-to-equator surface temperature gradient. Figure 2 shows how increases in the meridional insolation gradient $\left(\Delta_{\mathrm{s}}\right)$ lead to increases in meridional temperature gradients and extratropical static stability. The global-mean surface air temperature (the temperature at the lowest model level) changes by less than $8 \mathrm{~K}$ over this second series of simulations, compared with a change of $56 \mathrm{~K}$ resulting from changes in longwave optical thickness in the first series of simulations.

\section{Behavior of eddy kinetic energy}

The total (column integrated) EKE and other energies are shown in Figs. 3 and 4 for the two series of 
a

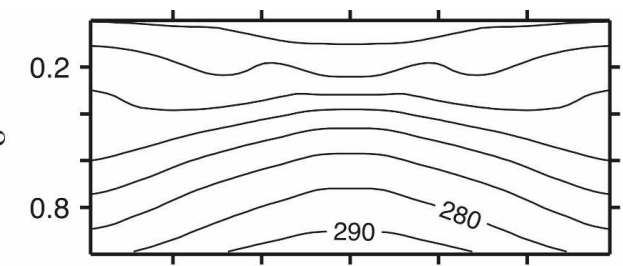

b

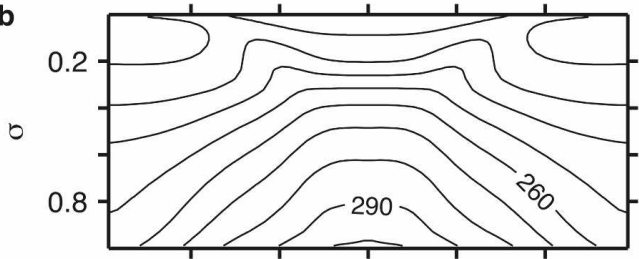

c

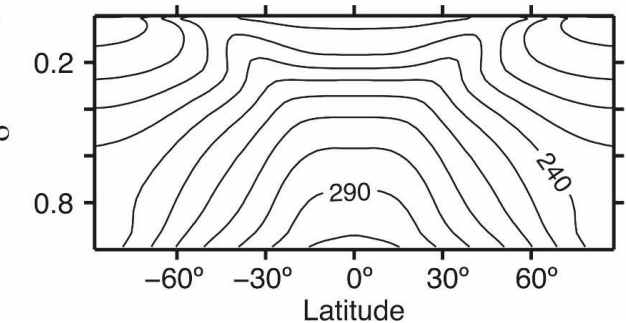

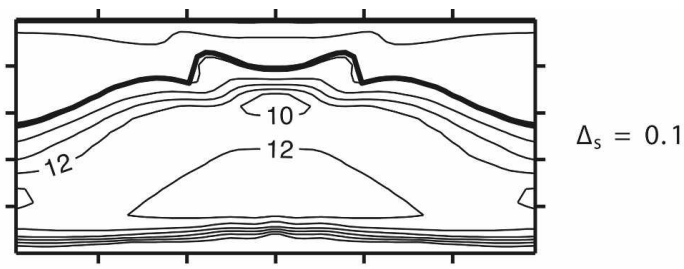

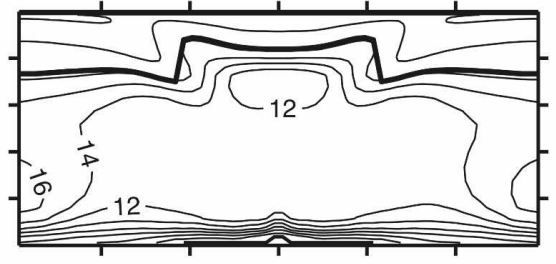

$\Delta_{\mathrm{s}}=1.2$

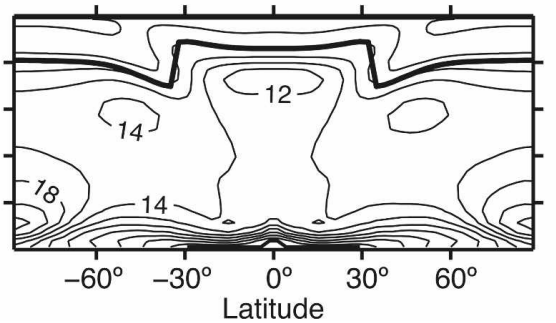

$\Delta_{\mathrm{s}}=1.95$

FIG. 2. As in Fig. 1 but for simulations in the series in which the meridional insolation gradient is varied $(\alpha=1)$ : (a) simulation with the smallest insolation gradient $\left(\Delta_{\mathrm{s}}=0.1\right),(\mathrm{b})$ reference simulation $\left(\Delta_{\mathrm{s}}=1.2\right)$, and (c) simulation with the largest insolation gradient $\left(\Delta_{\mathrm{s}}=1.95\right)$. The reference simulation is shown both here and in Fig. 1 to facilitate comparison with the other simulations.

simulations. For the series in which the longwave optical thickness is varied, results are plotted against the global-mean surface air temperature, which increases with increasing longwave optical thickness. For the series in which the meridional insolation gradient is varied, results are plotted against the mean surface air temperature contrast between pole and equator, which increases with increasing insolation gradient. Each circle represents the statistically steady state of a simulation. The energies shown are averaged over the baroclinic zones in both, statistically identical hemispheres. The baroclinic zones are defined as the regions within $15^{\circ}$ latitude of the maximum of the vertically integrated eddy potential temperature flux $\overline{v^{\prime} \theta^{\prime}} \cos \phi$, where the vertical integral is performed with respect to $\sigma$ from the surface to the lowest level of the tropopause. ${ }^{1}$ Eddy quantities $(\cdot)^{\prime}$ are defined relative to a surface-pressure-weighted zonal and time mean $\overline{(\cdot)}$ along $\sigma$ surfaces, and $v$ is meridional velocity, $\theta$ potential temperature, and $\phi$ latitude. The choice of averaging conven-

\footnotetext{
${ }^{1}$ The tropopause is determined as a level with zonal- and timemean temperature lapse rate of $2 \mathrm{~K} \mathrm{~km}^{-1}$. In the coldest simulation, the tropopause becomes poorly defined in high latitudes; these latitudes are excluded from the calculation of the lowest level of the tropopause for this simulation (cf. Fig. 1a).
}

tion is not important for the scaling of EKE (global averages give similar behavior).

Figure 3 shows that the total EKE, as a function of the global-mean surface air temperature, varies non-

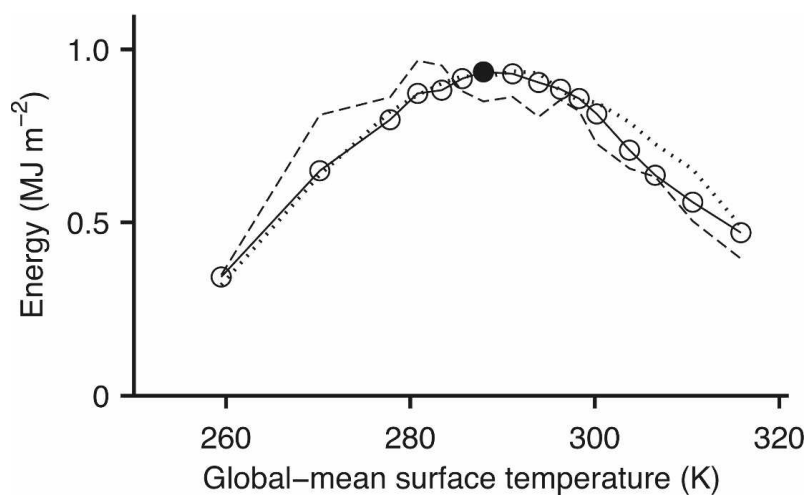

FIG. 3. Total EKE (solid line with circles), rescaled near-surface EKE (dotted line), and rescaled MAPE (dashed line) vs globalmean surface air temperature in the series of simulations in which the longwave optical thickness is varied. The filled circle indicates the reference simulation. All energies shown in this and subsequent figures are averaged over the baroclinic zones in both, statistically identical, hemispheres. Near-surface EKE is rescaled by a constant factor of 32 and MAPE is rescaled by a constant factor of 2.4. The rescaling factors are chosen using a least squares fit to a linear relationship through the origin with the total EKE, where data points from both series of simulations are included. 


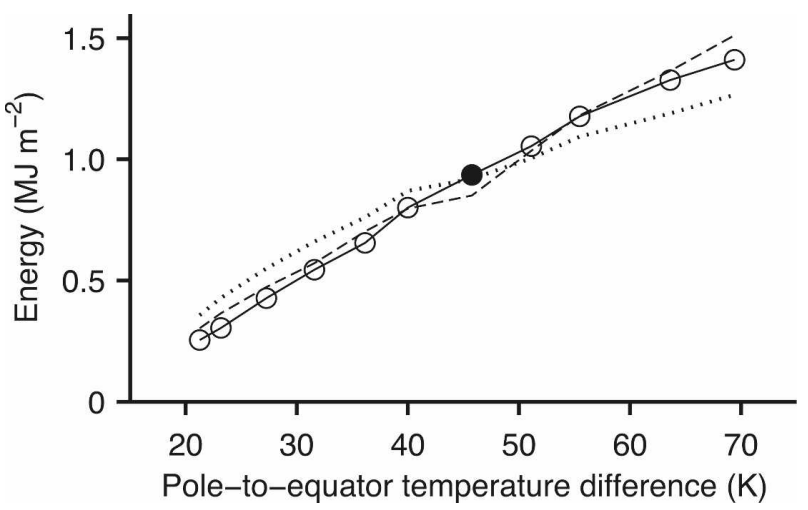

FIG. 4. As in Fig. 3 but for the series of simulations in which the meridional insolation gradient is varied. The energies are plotted against the mean surface air temperature contrast between pole and equator. The rescaling factors for near-surface EKE and MAPE are the same as in Fig. 3.

monotonically as the longwave optical thickness is varied. The eddy kinetic energy is maximal for a climate close to the reference climate, which has a global-mean surface air temperature close to that of present-day earth. Because the reference climate is close to a maximum in eddy kinetic energy, the sensitivity of the eddy kinetic energy to changes in longwave optical thickness (with shortwave parameters held fixed) is relatively low for the reference climate.

Figure 4 shows that the total EKE increases with increasing pole-to-equator surface temperature contrast as the meridional insolation gradient is increased. The response to changes in insolation gradient is monotonic over a wide range of climates, in contrast to the response to changes in longwave optical thickness (Fig. 3).

Figures 3 and 4 also show the behavior of nearsurface EKE, defined as the EKE per unit area of the atmosphere below $\sigma=0.9$. In both series of simulations, near-surface EKE scales approximately linearly with total EKE, and so our results for the scaling of total EKE are also relevant for surface storminess.

The changes in magnitude of EKE are accompanied by a poleward movement of the baroclinic zones as the longwave optical thickness is increased: they move $15^{\circ}$ in latitude from the coldest to the warmest simulation. A poleward shift of the storm tracks is also seen in global warming simulations (Yin 2005; Bengtsson et al. 2006). The baroclinic zones move by less than $4^{\circ}$ as the meridional insolation gradient is varied.

\section{Scaling of eddy kinetic energy with mean available potential energy}

Because MAPE is defined in terms of the zonal- and time-mean thermal structure of the atmosphere, a re-

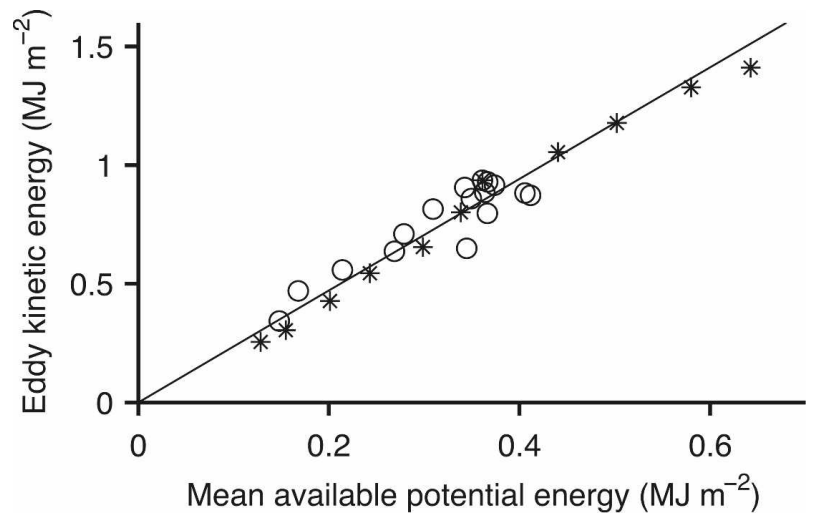

FIG. 5. Total EKE vs MAPE for all simulations. The series of simulations in which the longwave optical thickness is varied is shown with circles, and that in which the meridional insolation gradient is varied with asterisks. The solid line shows a linear relation through the origin with slope 2.4 (the rescaling factor used for MAPE in Figs. 3 and 4).

lationship between EKE and MAPE provides a basis for understanding how EKE changes in response to changes in radiative forcing. An approximate linear scaling relationship between EKE and MAPE is shown in Figs. 3 and 4, and more directly for both series of simulations in Fig. 5. The agreement with linear scaling is slightly better for changes in meridional insolation gradient than changes in longwave optical thickness. Nevertheless, Fig. 3 shows qualitatively similar behavior of MAPE and EKE as the longwave optical thickness is varied, with relatively little change in energy around the reference simulation, and smaller energies for very cold and very warm climates.

MAPE is calculated using the quadratic approximation of Lorenz (1955) in sigma coordinates, but excluding levels below $\sigma_{s}=0.9$ to avoid numerical problems with low static stability and excluding levels above the lowest level of the tropopause in the baroclinic zone. Use of the lowest level of the tropopause in the baroclinic zone (rather than, say, the mean level) helps exclude all contributions from the stratosphere. The resulting expression for MAPE per unit area is

$$
\text { MAPE }=\frac{c_{p} p_{0}}{2 g} \int_{\sigma_{t}^{\max }}^{\sigma_{s}} d \sigma \Gamma\left(\frac{\langle\bar{p}\rangle}{p_{0}}\right)^{\kappa}\left(\left\langle\bar{\theta}^{2}\right\rangle-\langle\bar{\theta}\rangle^{2}\right),
$$

where $\langle\cdot\rangle$ is the average over the baroclinic zone, $\bar{\theta}$ is the zonal- and time-mean potential temperature, $\theta_{t}^{\max }$ is the lowest level of the tropopause in the baroclinic zone, $\sigma_{S}=0.9$, and $p_{0}=10^{5} \mathrm{~Pa}$ is a reference surface pressure. The factor $\Gamma$ is an inverse measure of the dry static stability,

$$
\Gamma=-\frac{\kappa}{\langle\bar{p}\rangle}\left\langle\frac{\partial \bar{\theta}}{\partial p}\right\rangle^{-1}
$$


Other symbols have their usual meanings (e.g., Holton 2004).

A linear scaling between EKE and MAPE was found to hold over a wide range of dry simulations in Schneider and Walker (2008). ${ }^{2}$ We emphasize that even though our GCM includes moist processes, the MAPE we use does not explicitly take moist processes into account, and so, for example, the static stability used to calculate MAPE is the dry static stability. We also find linear scaling between EKE and the eddy available potential energy for the simulations presented here, but we focus on the scaling with MAPE because of its value in relating eddy to mean fields.

The scaling of MAPE (but not EKE) is sensitive to the horizontal average used. For example, use of a global average in the series in which the longwave optical thickness is varied results in relatively greater MAPE for the warmer simulations compared with the colder simulations. This seems to be related to a region of large meridional temperature gradients at upper levels close to the subtropical jet (cf. Fig. 1c, left panel). The scaling of MAPE is sensitive to whether or not this region is included, and thus to whether a global or baroclinic zone average is used. If a baroclinic zone average is used, there is also sensitivity to how the zone is defined. MAPE per unit area averaged over the baroclinic zone increases as the square of the width of the baroclinic zone, so that a wider baroclinic zone provides a greater reservoir of energy per unit area if the temperature gradient remains constant. Use of a baroclinic zone defined as in Schneider and Walker (2006; based on the latitudes at which the eddy potential temperature flux at $\sigma=0.84$ reaches a critical fraction 0.3 of its maximum value) gives similar results to those shown here. However, it is then difficult to explain the physical basis of the variation of the baroclinic zone width, and there is sensitivity to the sigma level and value of the critical fraction chosen. Use of a critical fraction of 0.7 , as in Schneider and Walker (2008), gives different results for some simulations. Because of these uncertainties, we instead average over a baroclinic zone of fixed width around the latitude of the maximum eddy potential temperature flux, as described in section 3. Use of somewhat different half widths (e.g., $10^{\circ}$ or $20^{\circ}$ for the baroclinic zones does not significantly change our results. We use the vertically averaged eddy potential temperature flux rather than the near-surface flux to define baroclinic zones because it gives slightly clearer

\footnotetext{
${ }^{2}$ Exceptions to linear scaling occurred for some simulations in which convection controlled the extratropical stratification. Eddies influence the extratropical stratification in all simulations presented here (cf. Schneider and O'Gorman 2008).
}

linear scaling of EKE with MAPE and because use of the near-surface flux is difficult to justify in some of the warmer simulations in which the maximum flux in the troposphere does not occur near the surface.

Given the level of accuracy of the linear scaling of EKE and MAPE and the uncertainties in the choice of averaging regions, we cannot exclude the possibility that latent heat release causes a deviation from linear scaling between EKE and MAPE. Our confidence in linear scaling is increased by results from a different set of simulations (not shown) in which the solar constant is varied rather than the longwave optical thickness. For these simulations, there is much less sensitivity to the choice of averaging regions, and the linear scaling between EKE and MAPE also holds over a wide range of simulations that includes very warm and moist climates.

\section{Factors affecting mean available potential energy}

To analyze the different factors contributing to MAPE changes, it is helpful to make an approximation of MAPE involving the mean meridional temperature gradient and static stability. Similar approximations of MAPE have been derived previously (e.g., Schneider 1981; Schneider and Walker 2008). We begin by approximating the potential temperature variance as

$$
\left\langle\bar{\theta}^{2}\right\rangle-\langle\bar{\theta}\rangle^{2} \sim\left\langle\partial_{y} \bar{\theta}\right\rangle^{2} L_{Z}^{2} / 12,
$$

where $y$ denotes meridional distance, and $L_{Z}$ is the meridional width of the baroclinic zone. Our choice of a fixed-width averaging region means that $L_{Z}$ is a constant. Approximating the vertical integral in (3) then leads to a scaling estimate for MAPE written in terms of the zonal- and time-mean temperature $\bar{T}$ as

$$
\text { MAPE } \sim \frac{c_{\mathrm{p}} p_{0}}{24 g}\left(\sigma_{s}-\sigma_{t}^{\max }\right) L_{Z}^{2}[\Gamma]_{v}\left[\left\langle\partial_{y} \bar{T}\right\rangle\right]_{v}^{2},
$$

where $[\cdot]_{v}$ is a vertical average from $\sigma_{t}^{\max }$ to $\sigma_{s}$.

The approximate MAPE (6) is shown in Figs. 6a and $7 \mathrm{a}$ and is adequately accurate for our purposes. In the following, we discuss its dependence on the meridional temperature gradient $\left[\left\langle\partial_{y} \bar{T}\right\rangle\right]_{v}$, inverse dry static stability $[\Gamma]_{v}$, and extratropical tropopause level $\sigma_{t}^{\max }$. The approximate MAPE scales like the vertically integrated Eady growth rate squared times the baroclinic zone width squared. This provides a link to studies that use the Eady growth rate to account for changes in the storm tracks (e.g., Geng and Sugi 2003; Yin 2005; Caballero and Langen 2005; $\mathrm{Li}$ and Battisti 2008). However, the Eady growth rate evaluated in the lower tro- 

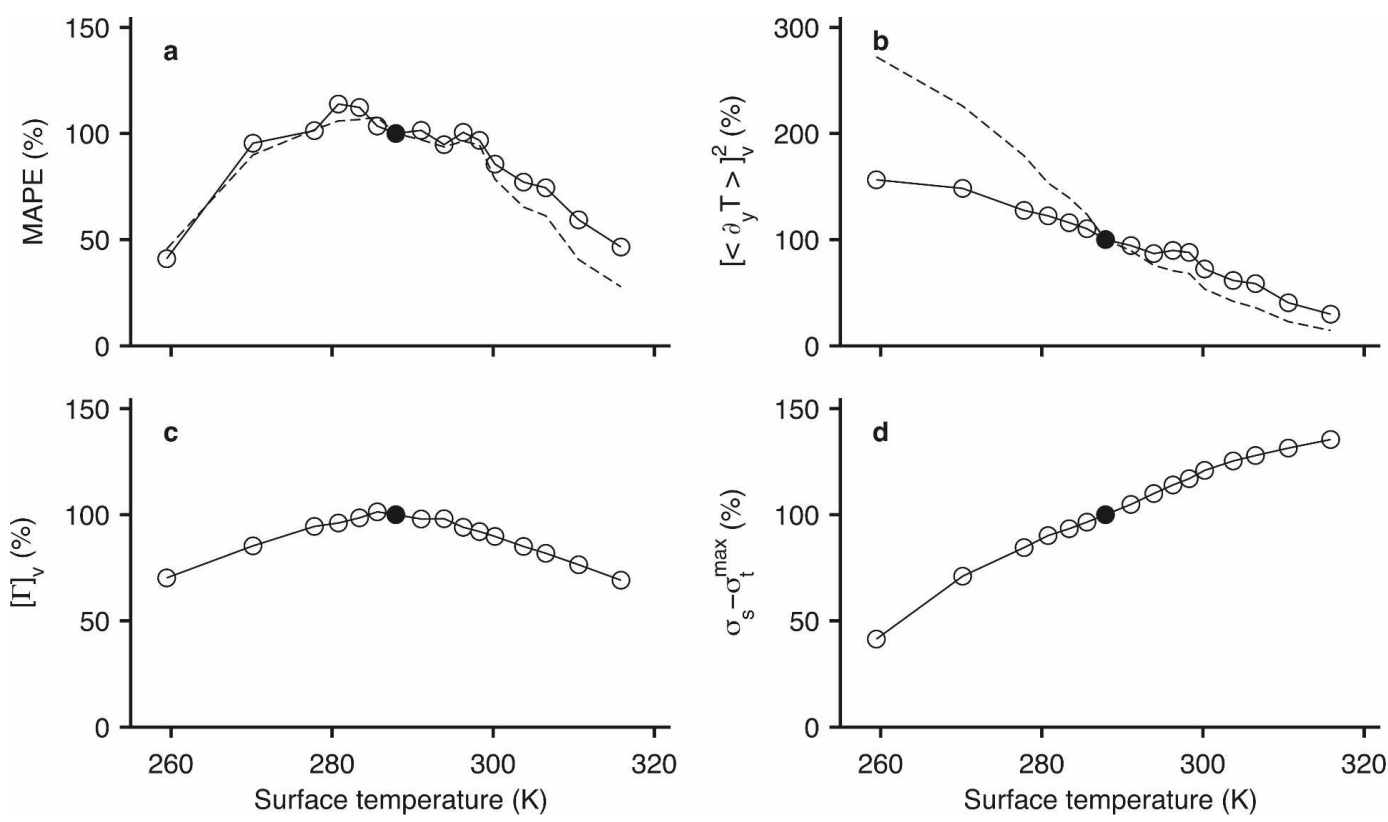

FIG. 6. Analysis of MAPE changes in terms of factors affecting the approximate MAPE (6) for the series of simulations in which the longwave optical thickness is varied. Results are plotted against the global-mean surface air temperature. All quantities are expressed as percentages of their reference simulation values: (a) MAPE (solid line with circles) and approximate MAPE (6) (dashed line). (b) Square of the vertically averaged meridional temperature gradient $\left[\left\langle\partial_{y} \bar{T}\right\rangle\right]_{v}^{2}$ (solid line with circles) and square of the meridional temperature gradient at the surface (dashed line). (c) Vertically averaged inverse dry static stability $[\Gamma]_{v^{*}}$ (d) Depth of the extratropical troposphere $\sigma_{s}-\sigma_{t}^{\max }$. The filled circles indicate the reference simulation. The values at the reference simulation are (a) $0.36 \mathrm{MJ} \mathrm{m}^{-2}$ for MAPE and $0.55 \mathrm{MJ} \mathrm{m}^{-2}$ for its approximation, (b) $2.6 \times 10^{-11} \mathrm{~K}^{2} \mathrm{~m}^{-2}$ for the vertical average and $2.9 \times 10^{-11} \mathrm{~K}^{2} \mathrm{~m}^{-2}$ for the surface quantity, (c) $7.5 \times 10^{-3} \mathrm{~K}^{-1}$, and (d) 0.60 .
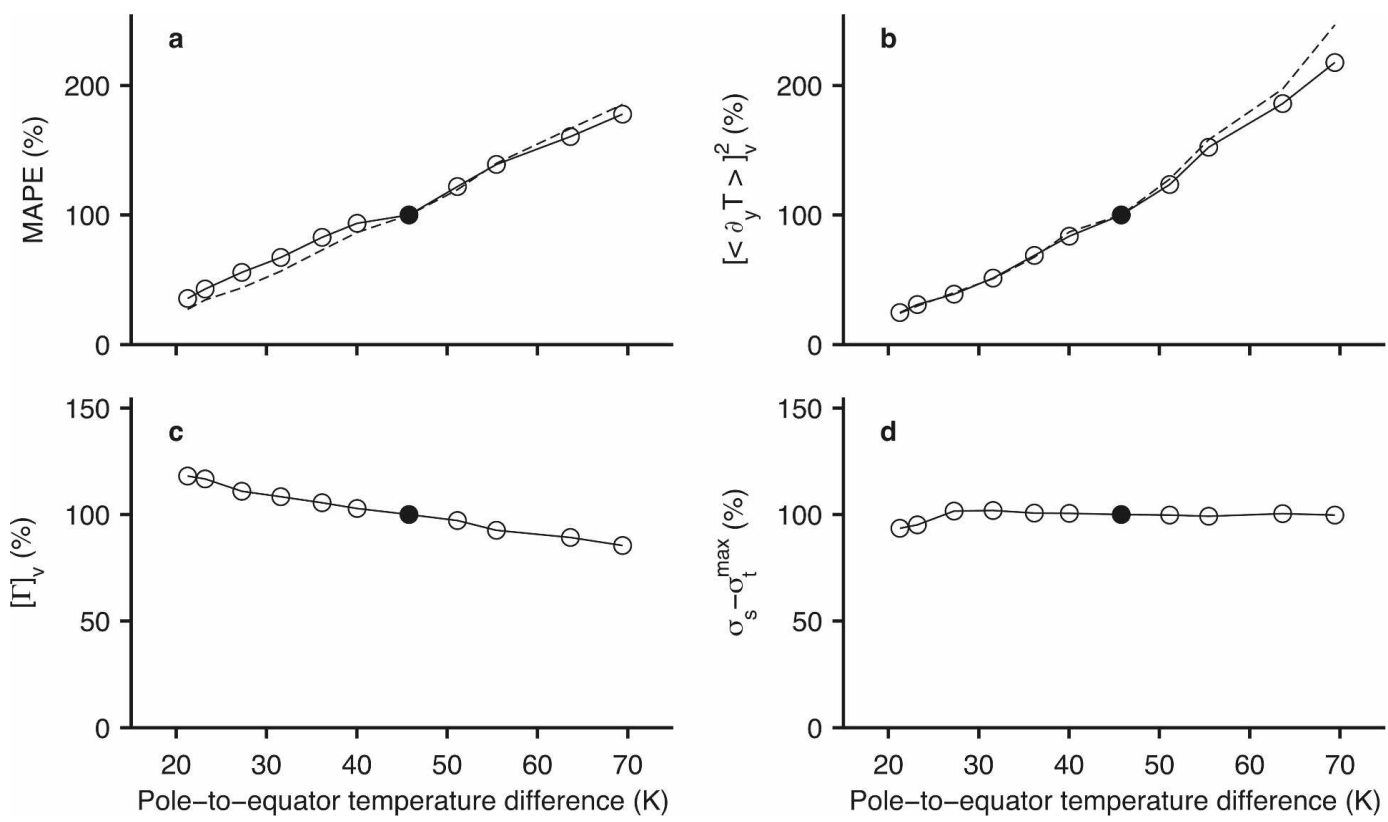

FIG. 7. As in Fig. 6 but for the series of simulations in which the meridional insolation gradient is varied.

Results are plotted against the mean surface air temperature contrast between pole and equator. 
posphere (e.g., Geng and Sugi 2003; Caballero and Langen 2005; Li and Battisti 2008) generally does not behave similarly to MAPE if there are changes in tropopause height or different changes in meridional temperature gradients at different levels (cf. Fig. 6).

A simpler scaling for the eddy velocity (related to the theory of Stone 1972) was used by Frierson et al. (2007) for simulations in which the effect of latent heating was varied in a GCM similar to the one used here. The simpler scaling depends on the meridional temperature gradient at one level and the Coriolis parameter at the latitude of the storm track, and it is adequate for the series of simulations in which the meridional insolation gradient is varied. However, in the form used in Frierson et al. (2007), it does not explicitly involve the tropopause pressure level or static stability. Such a scaling does not account for the eddy kinetic energy variations in the series of simulations in which the longwave optical thickness is varied but instead behaves more like the meridional temperature gradient (cf. Fig. 6b).

\section{a. Meridional temperature gradient}

The approximate MAPE (6) depends on the square of the meridional temperature gradient averaged over the depth of the troposphere. For the series of simulations in which the longwave optical thickness is varied, Fig. 6b indicates a decrease with increasing surface temperature of the vertically averaged temperature gradient $\left[\left\langle\partial_{y} \bar{T}\right\rangle\right]_{v}$; the surface temperature gradient decreases more strongly with increasing surface temperature (see also Fig. 1). The square of the vertically averaged temperature gradient decreases by a factor of 5.3 from the coldest to the warmest simulation, compared with a decrease by a factor of 19 in the square of the surface temperature gradient. The difference in the changes in meridional temperature gradients at different levels means that consideration of surface temperature gradients alone would lead to an overestimate of the effect of meridional temperature gradient changes on MAPE (and thus eddy kinetic energy). The difference in the response of the temperature gradient at the surface and in the upper troposphere can be understood in terms of the different changes in temperature lapse rate at low and high latitudes (Held 1978): with increasing temperature and increasing specific humidity, the lapse rate decreases in low latitudes (where it is close to the local moist adiabatic lapse rate), but increases, or decreases more slowly, in high latitudes (where it is more stable than moist adiabatic, but closer to moist adiabatic in warm climates; Schneider and O'Gorman 2008). The decrease in surface temperature gradient with increasing global-mean surface temperature shows that the sensitivity of surface temperature to changes in radia- tive forcing can be greater at high latitudes even without surface albedo feedbacks (cf. Held 1978). The poleward energy flux at $50^{\circ}$ increases as the surface temperature increases for all but the warmest simulations (O'Gorman and Schneider 2008), broadly consistent with the preferential warming of high latitudes implied by Fig. $6 b$.

The changes in meridional temperature gradients are more straightforward to understand for the series of simulations in which the meridional insolation gradient is varied (Fig. 7b). They account for most of the change in MAPE in this series of simulations (cf. Figs. 7a,b). The vertically averaged and surface meridional temperature gradients change in almost the same way, increasing by factors of 8.8 and 10, respectively, from the smallest to the largest insolation gradient. The effect of increasing the meridional insolation gradient is to increase meridional temperature gradients and thus MAPE.

\section{b. Dry static stability}

Greater extratropical dry static stability reduces the inverse stability $\Gamma$ and thus reduce MAPE. For the series of simulations in which the longwave optical thickness is varied, Fig. 6c shows that the simulation with maximum $\Gamma$ (minimum extratropical static stability) is close to the reference simulation. The average $\Gamma$ values of the coldest and warmest simulations are $30 \%$ and $31 \%$ smaller than that of the reference simulation (see also Fig. 1). Thus, for climates significantly warmer or colder than the reference climate, the extratropical dry static stability increases relative to the reference climate, with a consequent negative effect on MAPE. Greater extratropical static stability in colder climates is consistent with baroclinic eddies stabilizing the troposphere because the lower tropopause and greater meridional surface temperature gradient imply a stronger stabilizing effect of baroclinic eddies (Schneider and Walker 2006; Schneider and O'Gorman 2008). For warmer climates, moist convection and large-scale condensation become increasingly important for the stratification of the extratropics, and the dry static stability of moist adiabats increases. However, the analysis of Schneider and O'Gorman (2008) suggests that current theories do not account for the extratropical thermal stratification over the entire range of this series of simulations.

For the series of simulations in which the meridional insolation gradient is varied, the extratropical static stability increases monotonically with increasing pole-toequator temperature difference (Fig. 7c). This partially opposes the effects of the change in meridional temperature gradient on MAPE. The inverse stability $\Gamma$ 
decreases by a factor of 1.4 from the smallest to the largest insolation gradient. Qualitatively, such increases in static stability with increasing meridional temperature gradients are predicted by a number of theories of the extratropical thermal stratification (Stone 1978; Juckes 2000; Schneider and Walker 2006).

\section{c. Tropopause pressure}

For the series of simulations in which the longwave optical thickness is varied, Fig. $6 \mathrm{~d}$ shows that the tropopause pressure decreases (tropopause height increases; see Fig. 1) with increasing surface temperature, with a consequent positive effect on MAPE. The depth of the troposphere measured as $\sigma_{s}-\sigma_{t}^{\max }$ increases by a factor of 3.3 from the coldest to the warmest simulation. For a given lapse rate, the radiative constraint on tropopause height implies that the tropopause height increases with increasing longwave optical thickness because the surface temperature and emission height increase (Thuburn and Craig 1997, 2000; Schneider 2007). The lapse rate variations implied by Fig. $6 \mathrm{c}$ modulate this response.

For the series of simulations in which the meridional insolation gradient is varied, changes in tropopause pressure are relatively unimportant for the behavior of MAPE (Fig. 7d). The depth of the troposphere $\left(\sigma_{s}-\right.$ $\left.\sigma_{t}^{\max }\right)$ varies by less than $9 \%$ of its reference simulation value over this series of simulations.

\section{Conclusions}

We have investigated the response of EKE to changes in radiative forcing over a wide range of climates in a moist idealized GCM. We have discussed two series of simulations with relevance to past and projected climate changes: 1 ) a series in which changes in greenhouse gas concentrations are represented by changes in longwave optical thickness with shortwave parameters held constant and 2) a series in which changes in the distribution of absorbed solar radiation are represented by changes in meridional insolation gradient with longwave parameters held constant. The series of simulations in which the longwave optical thickness is varied demonstrates that dynamical processes can lead to a climatic maximum for EKE. The EKE is near a maximum for a climate with temperatures close to those of present-day earth. Because of the linear scaling between total and near-surface EKE, this also shows the potential for a climatic maximum in extratropical surface storminess. Such a maximum in EKE, if present in earth's climate system, may occur at a different global-mean temperature than our simulations because of processes not included here (e.g., high- latitude surface albedo feedbacks). Indeed, the second series of simulations suggests that one effect of decreases in high-latitude albedo (melting ice) would be a decrease in the pole-to-equator temperature contrast with a consequent negative effect on MAPE and EKE. It is possible that decreases in EKE played an important role in past warm and cold climates. For example, decreases in EKE in very warm climates may have partially offset the enhancement of the poleward energy flux owing to the greater moisture content of the atmosphere (Caballero and Langen 2005). It is also possible that the North Atlantic storm track was weaker at the Last Glacial Maximum ( $\mathrm{Li}$ and Battisti 2008).

We have shown that there is an approximate linear scaling between EKE and dry MAPE over a wide range of climates despite the presence of moist processes in our idealized GCM. Such a scaling is of use in understanding the order of magnitude and sign of changes in EKE in response to climate changes. Uncertainties in the appropriate definition of the baroclinic zones over which to average are a weakness of this result, a problem that might be alleviated by simulating moist baroclinic eddies using a primitive equation model in a simpler geometry such as a channel.

Using a simple approximation, we have described the dependence of MAPE on dry static stability, meridional temperature gradients, and tropopause pressure level. MAPE depends on the meridional temperature gradient averaged over the depth of the troposphere. For the series of simulations in which the longwave optical thickness is varied, the vertically averaged temperature gradient is not as sensitive to climate change as the surface temperature gradient, although its changes are nonetheless significant for the behavior of the MAPE. The relative insensitivity of MAPE to longwave optical thickness changes near the reference simulation arises because of opposing changes in the tropopause height and meridional temperature gradients at different levels, combined with a local minimum in static stability. Temperature gradients and static stabilities throughout the depth of the troposphere affect MAPE and hence the surface storminess as measured by near-surface EKE.

Our study offers a basis for understanding changes in eddy energies in the extratropical storm tracks in more complex atmospheric flows, provided that the approximate linear scaling between EKE and MAPE continues to hold, and that the dynamical changes in static stability and meridional temperature gradients that we have discussed indeed occur. Mesoscale effects of latent heat release on storm intensity are not resolved in our simulations and could change significantly with climate, affecting, for example, wind speed extremes. 
Acknowledgments. We are grateful for support by the National Science Foundation (Grant ATM450059), the Davidow Discovery Fund, and a David and Lucile Packard Fellowship. The simulations were performed on Caltech's Division of Geological and Planetary Sciences Dell cluster. We thank Dargan Frierson for providing code for the convection and radiation schemes and Chris Walker for development of the postprocessing code.

\section{REFERENCES}

Bengtsson, L., K. I. Hodges, and E. Roeckner, 2006: Storm tracks and climate change. J. Climate, 19, 3518-3543.

Caballero, R., and P. L. Langen, 2005: The dynamic range of poleward energy transport in an atmospheric general circulation model. Geophys. Res. Lett., 32, L02705, doi:10.1029/ 2004GL021581.

Chang, E. K. M., S. Lee, and K. L. Swanson, 2002: Storm track dynamics. J. Climate, 15, 2163-2183.

Emanuel, K. A., M. Fantini, and A. J. Thorpe, 1987: Baroclinic instability in an environment of small stability to slantwise moist convection. Part I: Two-dimensional models. J. Atmos. Sci., 44, 1559-1573.

Frierson, D. M. W., 2007: The dynamics of idealized convection schemes and their effect on the zonally averaged tropical circulation. J. Atmos. Sci., 64, 1959-1976.

— I. M. Held, and P. Zurita-Gotor, 2007: A gray-radiation aquaplanet moist GCM. Part II: Energy transports in altered climates. J. Atmos. Sci., 64, 1680-1693.

Geng, Q., and M. Sugi, 2003: Possible change of extratropical cyclone activity due to enhanced greenhouse gases and sulfate aerosols-Study with a high-resolution AGCM. J. Climate, 16, 2262-2274.

Green, J. S. A., 1970: Transfer properties of the large-scale eddies and the general circulation of the atmosphere. Quart. J. Roy. Meteor. Soc., 96, 157-185.

Gutowski, W. J., Jr., L. E. Branscome, and D. A. Stewart, 1992: Life cycles of moist baroclinic eddies. J. Atmos. Sci., 49, 306319.

Held, I. M., 1978: The tropospheric lapse rate and climatic sensitivity: Experiments with a two-level atmospheric model. $J$. Atmos. Sci., 35, 2083-2098.

_ 1993: Large-scale dynamics and global warming. Bull. Amer. Meteor. Soc., 74, 228-241.

Holton, J. R., 2004: An Introduction to Dynamic Meteorology. 4th ed. Elsevier Academic Press, 535 pp.

Juckes, M. N., 2000: The static stability of the midlatitude troposphere: The relevance of moisture. J. Atmos. Sci., 57, 30503057.

Korty, R. L., and T. Schneider, 2007: A climatology of the tropo- spheric thermal stratification using saturation potential vorticity. J. Climate, 20, 5977-5991.

Lambert, S. J., 1995: The effect of enhanced greenhouse warming on winter cyclone frequencies and strengths. J. Climate, $\mathbf{8}$, $1447-1452$.

Lapeyre, G., and I. M. Held, 2004: The role of moisture in the dynamics and energetics of turbulent baroclinic eddies. J. Atmos. Sci., 61, 1693-1710.

Li, C., and D. S. Battisti, 2008: Reduced Atlantic storminess during Last Glacial Maximum: Evidence from a coupled climate model. J. Climate, 21, 3561-3579.

Lorenz, E. N., 1955: Available potential energy and the maintenance of the general circulation. Tellus, 7, 157-167.

, 1979: Numerical evaluation of moist available energy. Tellus, 31, 230-235.

Meehl, G. A., and Coauthors, 2007: Global climate projections. Climate Change 2007: The Physical Science Basis, S. Solomon et al., Eds., Cambridge University Press, 747-846.

O'Gorman, P. A., and T. Schneider, 2008: The hydrological cycle over a wide range of climates simulated with an idealized GCM. J. Climate, 21, 3815-3832.

Peixoto, J. P., and A. H. Oort, 1992: Physics of Climate. American Institute of Physics, $520 \mathrm{pp}$.

Reed, R. J., A. J. Simmons, M. D. Albright, and P. Undén, 1988: The role of latent heat release in explosive cyclogenesis: Three examples based on ECMWF operational forecasts. Wea. Forecasting, 3, 217-229.

Schneider, E. K., 1981: On the amplitudes reached by baroclinically unstable disturbances. J. Atmos. Sci., 38, 2142-2149.

Schneider, T., 2007: The thermal stratification of the extratropical troposphere. The Global Circulation of the Atmosphere, T. Schneider and A. H. Sobel, Eds., Princeton University Press, 47-77.

, and C. C. Walker, 2006: Self-organization of atmospheric macroturbulence into critical states of weak nonlinear eddyeddy interactions. J. Atmos. Sci., 63, 1569-1586.

, and P. A. O'Gorman, 2008: Moist convection and the thermal stratification of the extratropical troposphere. J. Atmos. Sci., 65, 3571-3583.

, and C. C. Walker, 2008: Scaling laws and regime transitions of macroturbulence in dry atmospheres. J. Atmos. Sci., 65, 2153-2173

Stone, P. H., 1972: A simplified radiative-dynamical model for the static stability of rotating atmospheres. J. Atmos. Sci., 29, 405-418. , 1978: Baroclinic adjustment. J. Atmos. Sci., 35, 561-571.

Thuburn, J., and G. C. Craig, 1997: GCM tests of theories for the height of the tropopause. J. Atmos. Sci., 54, 869-882.

height: The radiative constraint. J. Atmos. Sci., 57, 17-28.

Yin, J. H., 2005: A consistent poleward shift of the storm tracks in simulations of 21 st century climate. Geophys. Res. Lett., 32, L18701, doi:10.1029/2005GL023684. 